\title{
High-Resolution Fingerprint Matching using Level 3 Incipient Ridges and Scars
}

\author{
V. Latha Jothi \\ Research Scholar \& AP(Sr.Gr.) \\ Department of Computer Science \& \\ Engineering, \\ Velalar College of Engineering and \\ Technology, \\ Erode - 638012, Tamilnadu, India
}

\author{
S. Arumugam, PhD. \\ Chief Executive Officer \\ Nandha Engineering College \\ Perundurai, Erode - 638052, \\ Tamilnadu, India
}

\begin{abstract}
Biometrics consists of automated methods for identifying and recognizing a person which can be either in the form of physiological or behavioral traits. Some of the features being considered are face, fingerprints, hand geometry, handwriting and voice. Fingerprint friction ridge details are hierarchically divided into 3 different levels which ranges from pattern (Level1) to minutia points (Level 2) and to pores and ridge contours (Level 3). Latent print examiners frequently use Level 3 features for finger print recognition and identification. But the Federal Bureau of Investigation's (FBI) standard for finger print resolution for Automated Fingerprint Identification Systems (AFIS) is 500 pixels per inch which is highly inadequate for capturing level 3 features. Increasing the scan resolution alone does not increase the performance of the system. In the proposed work incipient ridges and scars is extracted using Gabor filter technique which is combined with pores and ridges of Fingerprint. Geometric matching is performed for the level 3 features. Experiments conducted shows that the performance gain is $90 \%$ and error rate is $2.4571 \%$ for the proposed model which outperforms the existing work with level 3 features: ridges and pores.
\end{abstract}

\section{General Terms}

Fingerprint Biometrics, Level 3 feature recognition.

\section{Keywords}

Fingerprints, Automated Fingerprint Identification Systems, Incipient Ridges, scars, pores, ridges.

\section{INTRODUCTION}

Personal identification is the process of identifying the identity of an individual. The summarization of identity of a person can be divided into two different types of problems. They are personal identification and verification. Increase in the level of security rift prerequisite the need for secure identification and personal verification to become more vital.

Biometrics uses one of the most traditional systems to identify and monitor the people through unforgettable and unchanging physical characteristics. In today's scenario due to the advancement in modern technology the system is larger enough to guide and help us or even relieve us from the drudgery of recognition tasks.

Single biometrics does not satisfy the needs of all identification processes. Several number of biometrics patterns have been proposed and measured for the purpose of identification. Each biometrics features have its own advantages and disadvantages and based on it each biometric pattern and feature make it way for a specific identification application. The need for biometrics can be detected apparently in several state and local governments and found in the military. Some of the enterprise which are using predominantly are government organizations, e-banking, measuring and validating financial transactions, health factors and so on. Biometrics includes various parts of the body ranging from fingerprint to eye. Subsequently, the Federal Bureau of Investigation uses the fingerprints from a crime patrol in order to detect and identify a criminal involved in crime.

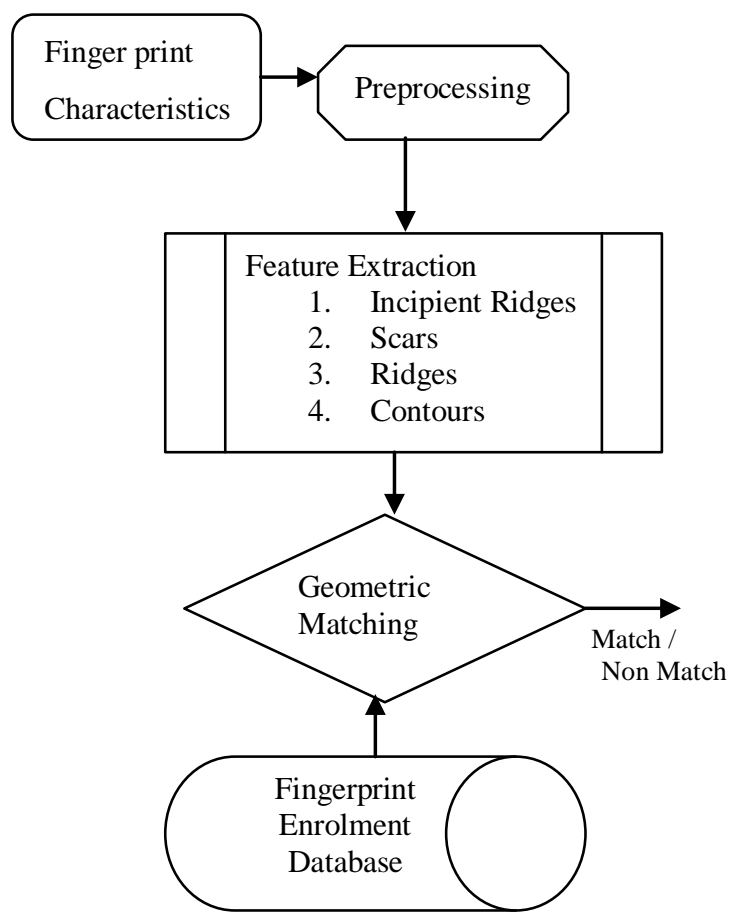

Figure1 : Framework of Fingerprint Identification System

Fingerprint identification has extensively used as a key to biometric technology in many criminal departments and forensic areas, to provide in with physical access control with a very higher degree of confidence. Figure 1 shows the framework of fingerprint identification system using the proposed features incipient ridges, scars and ridges and contours. Geometric matching is performed for the identification of fingerprints of an individual.

The work is organized as follows. In Section II related work is carried out. Section III introduces and analyzes the proposed fingerprint identification features extracted from the incipient 
ridge and scars. Fingerprint geometric matching algorithm with the help of incorporating conventional ridges and contours is also explained in Section III. Experimental results are conducted and shown in Section IV and Section V discusses with the conclusions.

\section{RELATED WORK}

The most widely used features in biometrics research studies is the fingerprint of an individual. Biometric process consists of considering various traits. For more than a century fingerprint matching has been extensively used for the sake of law enforcement. In Automated Fingerprint Recognition System (AFRS) [1] some form of ridges and valleys are noted on the skin of our palms. These ridges called as friction ridges which helps the hand to grasp the objects.

A new mode of fingerprint matching algorithm is proposed [6] where the ridge features are divided into four elements. The elements are ridge count, ridge length, ridge curvature direction and ridge type. The work proposes a novel matching mechanism by using Breadth First Search (BFS).

A filter-based algorithm [3] which uses Gabor filters to capture both local and global details in a fingerprint as a compact fixed length FingerCode. The fingerprint matching is based on the Euclidean distance between the two corresponding FingerCodes and hence is extremely fast.

An alignment-based elastic matching algorithm [2] is quite fast, because it is capable of finding the correspondences between minutia points without resorting to an exhaustive search. At the same time, this matching algorithm has a good performance, because it has the ability to adaptively compensate for the nonlinear deformations and inexact pose transformations between different fingerprints.

The performance of a fingerprint recognition system is heavily affected by the quality of fingerprint images [5], [8]. The highest improvement when rejecting low-quality samples is obtained for the false rejection rate at a given false acceptance rate [4].

A novel approach based on artificial neural networks for purpose of generating and implementing one biometric feature (faces) from another (only fingerprints) [7] is the new proposed system that generates all parts of the face including eyebrows, eyes, nose, mouth, ears and face border from only fingerprints.

\section{FINGERPRINT RECOGNITION USING INCIPIENT RIDGES AND SCARS (FRIRS)}

The proposed work make extensive use of Level 3 features (Incipient Ridges and Scars) along with the features Pores and Ridges for matching 1000 pixels per inches. A block diagram representing the process of fingerprint recognition using Incipient Ridges and Scars is given in fig 3.1

\subsection{Framework of FRIRS}

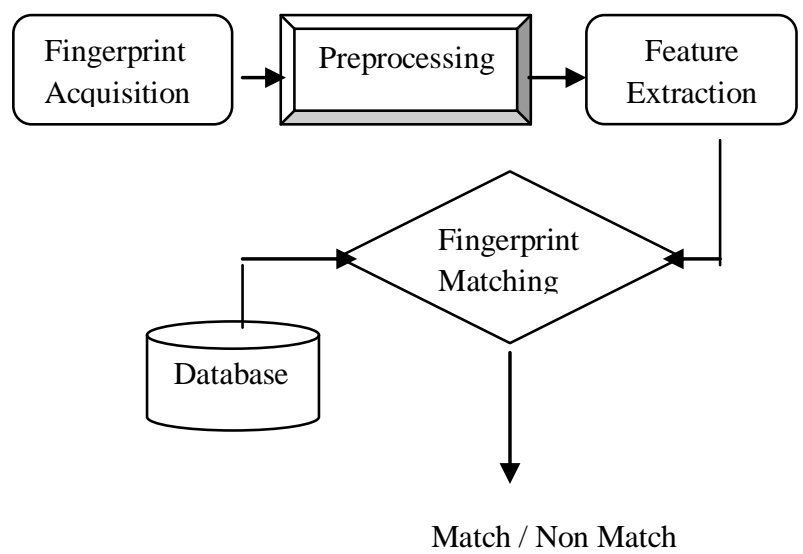

Figure 2: System Framework of FRIRS

The different phases involved in high resolution FRIRS is elaborated in Figure 2. The overall framework can be divided into four phases. They are:

1) Fingerprint Acquisition Phase

2) Preprocessing Phase

3) Feature Extraction Phase

4) Fingerprint Matching Phase.

The explanation of four phases involved in FRIRS is given below.

\subsubsection{Fingerprint Acquisition Phase}

Fingerprint recognition is an extensively used factor for the sake if identification and authentication of biometric traits. The first phase involved in FRIRS is the Fingerprint Acquisition Phase. This phase deals with the acquiring of fingerprint either using sensor device or from a group of fingerprints stored in the form of templates. Subsequently the images of fingerprints are fed into the database. Our work acquires the finger print from the templates.

\subsubsection{Preprocessing Phase}

The second phase in FRIRS is the Preprocessing Phase. The fingerprint image acquired from fingerprint acquisition phase undergoes a preprocessing for the sake of producing quality images. Low quality images and images with noise during fingerprint recognition make the matching phase a complicated task. The Preprocessing phase involved in FRIRS results in noise-free image which in turn produces high quality and accuracy. The purpose of performing the preprocessing stage is it to enhance the image that avoids the undesired distortions which are highly required for further processing stages. The steps involved in preprocessing phase are:
a) Histogram Equalization for Fingerprint
b) Filtering the equalized images 


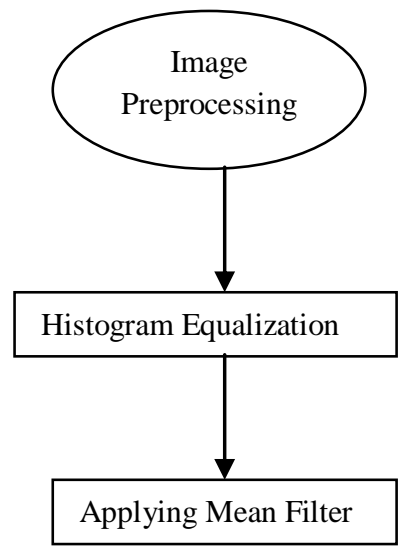

Figure 3: Image Preprocessing Phase

Figure 3 shows the steps involved in image preprocessing phase for finger print recognition. Raw fingerprint images cannot be further used until it has performed a preprocessing phase. The preprocessing phase involved in FRIRS is depicted in Figure 3. It consists of applying histogram equalization and applying mean filter. Histogram equalization for fingerprint is a procedure for adjusting image intensities to increase the contrast value. Let $S$ be the image of finger print represented by a matrix of integer pixel intensities ranging to 1000 pixels per inches for 256 pixels represented by $\mathrm{P}$. The normalized histogram for fingerprint is expressed as given below:

$\mathrm{H}_{\mathrm{n}}=$ Number of pixels for fingerprint with $\mathrm{n}$ intensity

Total number of pixels for fingerprint

The histogram image for fingerprint recognition is given by

$$
\mathrm{G}_{\mathrm{ij}}=\operatorname{floor}(\mathrm{P}-1) \sum_{\mathrm{n}=0}^{\mathrm{S}_{\mathrm{ij}}} \mathrm{H}_{\mathrm{n}}
$$

The main concept behind the median filtering for obtaining the equalized images is to run the process entry by entry and replacing each entry based on the neighboring entries. Implement the median filtering by using the following equation

$$
\mathrm{RI}=\mathrm{MF}\left(\mathrm{G}_{\mathrm{ij}}\right)
$$

\subsubsection{Feature Extraction Phase}

The Feature Extraction phase for fingerprint recognition combines the process of extracting incipient ridges, scars, ridges and contours using Gabor Filter process. Initially the image performs the enhancement process using Gabor filter. Subsequently the wavelet transform is applied to the image in order to extract the four features. The Gabor filter is of the form:

$\mathrm{F}(\mathrm{a}, \mathrm{b}: \lambda, \Omega, \mu, \sigma, \rho)=\frac{\exp \left(\mathrm{a}^{2}+\rho^{2} \mathrm{~b}^{2}\right)}{2 \sigma^{2}} * \exp \left(\mathrm{i}\left(2 \Pi \frac{(\mathrm{a})}{\lambda}+\mu\right)\right.$

Where $\lambda$ denotes the wavelength factor, $\Omega$ represents orientation format, $\mu$ denotes phase offset value, $\sigma$ represents sigma value and, $\rho$ denotes spatial aspect.

\subsubsection{Finger Print Matching Phase}

In the final phase is the Finger Matching phase which considers Minutia based matching process. The structure is in the form of star. This Minutia based Matching process consists of central point $C_{i}$ and its neighboring points defined by $\left(\mathrm{C}_{\mathrm{i} 1}, \mathrm{C}_{\mathrm{i} 2}, \mathrm{C}_{\mathrm{i} 3}, \ldots \mathrm{C}_{\mathrm{ik}}\right)$. The distance defined is $\mathrm{D}_{\max }$ from the central point $\mathrm{C}_{\mathrm{i}}$. The matching process for FRIRS matches with the central minutia points till a perfect match is obtained.

\subsection{Proposed Algorithm FRIRS}

Step 1: Register Fingerprint Images from CASIA Fingerprint Image Database Version 5.0.

Step 2: Perform preprocessing of fingerprint images using Histogram Equalization and Median Filtering.

Step 3: Obtain the feature using Gabor Filter and Wavelet Transform.

Step 4: Verify the fingerprint by performing Minutia based matching process.

\section{EXPERIMENTAL RESULTS AND DISCUSSION}

High-Resolution Fingerprint Matching is implemented using our proposed algorithm FRIRS. Performance of the proposed algorithm is evaluated using FRIRS. The new algorithm FRIRS was compared against our existing work which used ridges and pores as the feature. Geometric matching was performed for the features ridges and pores which is evaluated to $80 \%$ whereas our proposed work FRIRS achieves $90 \%$. The experimental results and discussion for FRIRS is explained as below. Experimental result conducted proves that this algorithm FRIRS proves to be efficient when compared to our existing algorithm FRPR.

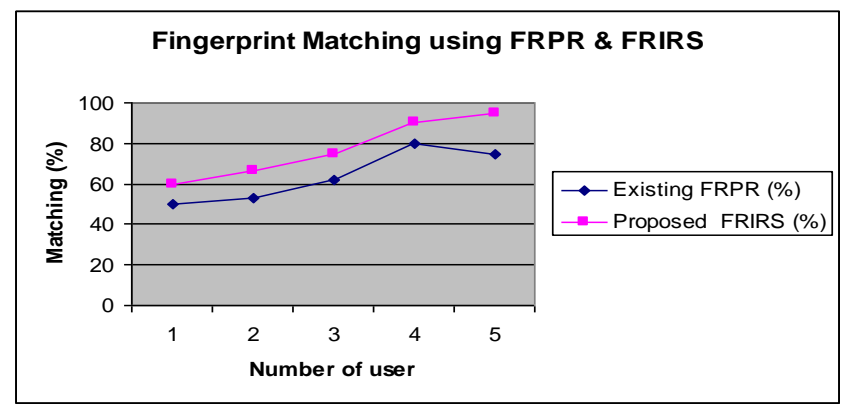

Figure 4: Matching (\%) for FRPR \& FRIRS

Figure 4 shows the performance of general matching work done for the existing FRPR and proposed FRIRS. In order to demonstrate effectiveness of the proposed work FRIRS, we have plotted the number of user and matching in terms of \&. The general matching curve for proposed work is plotted for number of user versus matching $(\%)$ for different users. It is observed from the graph that matching (\%) obtained for FRIRS is higher when compared to FRPR.

Figure 5 shows the Performance Gain of Fingerprint Recognition using FRPR and proposed algorithm FRIRS. The significant value of performance gain was noted for different quality fingerprint images. Due to the iterative mode of ICP algorithm involved in the existing work FRPR, it proved to be inefficient.

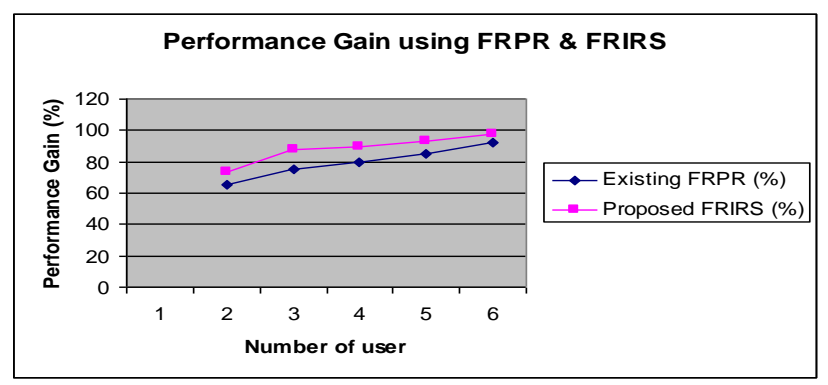

Figure 5: Performance Gain of the Proposed Algorithm 
By using Level 3 features as incipient ridges, scars, ridges and contours, our proposed work proves to get higher value of performance gain. From the graph it is clear that the performance gain of proposed work FRIRS is higher when compared to existing work FRPR.

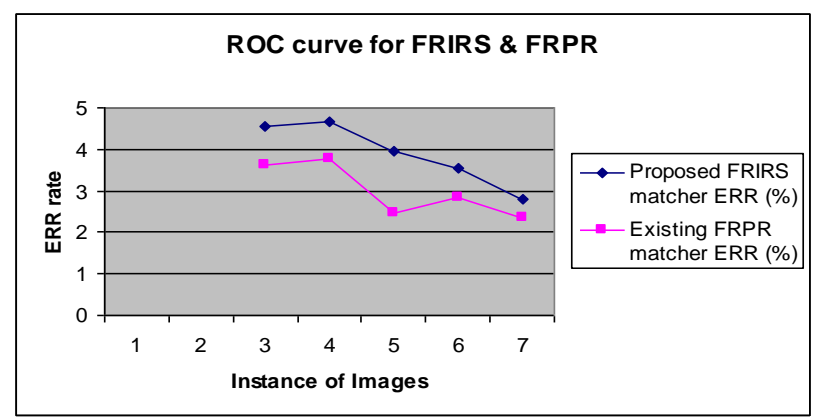

Figure 6: ROC curve for FRIRS \& FRPR

Figure 6 shows the ROC curve for Error Rate comparison between proposed FRIRS and existing FRPR algorithms. The aim of proposed work FRIRS is to minimize the error rate in terms of False Acceptance Rate and False Rejection Rate. The above experiments conducted proves that Level 3 features like incipient ridges, scars, ridges and contours involved in FRIRS minimizes the error rate. From the graph it is evidence that the proposed FRIRS shows better results when compared to FRPR. Increase in the number of comparisons results in decrease in the error rate.

\section{CONCLUSION}

The high resolution finger print matching for Level 3 features using incipient ridges, scars, pores and ridge contours is presented in this work. It produces higher performance gain with reduced error rate. The experimental result shows that FRIRS achieves $90 \%$ of performance gain which is higher than the existing model which achieves only $80 \%$. In addition, the error rate for FRIRS is $2.45 \%$ which is less than the existing work which is $3.93 \%$. The use of integrated Level 3 features: incipient ridges, scars with ridges and pores minimize the error rates like FAR and FRR. Optimization of the feature lists is obtained. Finally matching process is performed using Minutia matching with test and training images to enhance the capability of the work. It results in higher performance gain and low error rate.

\section{REFERENCES}

[1] Anil K. Jain, Jianjiang Feng, Karthik Nandakumar, "Fingerprint Matching", The IEEE Computer Society, pp. $36-44$, February 2010.

[2] Anil K Jain, Lin Hong, Ruud Bolle, "On-Line Fingerprint Verification", IEEE Transactions On Pattern Analysis And Machine Intelligence, Vol. 19, No. 4, pp. 302 - 314, April 1997

[3] Anil K. Jain, Salil Prabhakar, Lin Hong, and Sharath Pankanti, "Filterbank-Based Fingerprint Matching", IEEE Transactions on Image Processing, Vol. 9, No. 5, pp. 846 - 859, May 2000.

[4] Fernando Alonso-Fernandez, Julian Fierrez, Javier Ortega-Garcia, Joaquin Gonzalez-Rodriguez, Hartwig Fronthaler, Klaus Kollreider, and Josef Bigun, "A Comparative Study of Fingerprint Image-Quality Estimation Methods", IEEE Transactions on Information Forensics and Security, Vol. 2, No. 4, pp. $734-743$, December 2007.

[5] Fierrez-Aguilar. J, Chen.Y, Ortega-Garcia. Jain.A, "Incorporating image quality in multi-algorithm fingerprint verification", International Conference on. Biometrics, Vol. 3832, pp. 213-220, 2006 Springer.

[6] Heeseung Choi, Kyoungtaek Choi, and Jaihie Kim, "Fingerprint Matching Incorporating Ridge Features With Minutiae" IEEE Transactions on Information Forensics and Security, Vol. 6, No. 2, pp. 338 - 345, June 2011.

[7] Necla Ozkaya, and Seref Sagiroglu, "Generating One Biometric Feature from Another: Faces from Fingerprints" ISSN 1424-8220 Sensors 2010, 10, 42064237; doi:10.3390/s100504206.

[8] Simon-Zorita.D, Ortega-Garcia et al., "Image Quality and Position Variability Assessment in Minutiae-based Fingerprint Verification" IEEE Proceedings on Vision Image and Signal Processing, Vol. 150, No. 6, pp. 402 408, December 2003. 